\title{
Current status of the treatment of microscopic polyangiitis and granulomatosis with polyangiitis in Japan
}

\author{
Koichi Sugiyama $\cdot$ Ken-ei Sada $\cdot$ Michiko Kurosawa $\cdot$ \\ Jun Wada $\cdot$ Hirofumi Makino
}

Received: 23 August 2011/Accepted: 28 May 2012/Published online: 5 July 2012

(C) The Author(s) 2012. This article is published with open access at Springerlink.com

\begin{abstract}
Background This study aimed to describe the epidemiologic characteristics of microscopic polyangiitis (MPA) and granulomatosis with polyangiitis (GPA) in Japan.

Methods We used the database of the Ministry of Health, Labour and Welfare (MHLW) from 2006 to 2008, and analyzed data from 938 patients $(\mathrm{MPA}=697, \mathrm{GPA}=241$ ) who fulfilled the MHLW diagnostic criteria and had registered within a year after onset.

Results The mean ages of the MPA and GPA patients were $69.4 \pm 0.4$ and $58.4 \pm 1.1$ years, respectively. Renal $(86.9 \%)$, chest $(73.7 \%)$, and nervous system (45.2\%) symptoms were common in MPA patients. Ear, nose, and throat $(86.7 \%)$, chest $(78.0 \%)$, and renal (60.6 \%) symptoms were frequently observed in GPA patients. The concomitant use of cyclophosphamide (CY) with corticosteroids was observed in $22.2 \%$ of the MPA patients and $58.5 \%$ of the GPA patients. In multivariate analysis, the concomitant use of CY was associated with a younger age and pulmonary hemorrhage in MPA patients, and the avoidance of CY was associated with nervous system symptoms and rapidly progressive glomerulonephritis in GPA patients. Plasma exchanges were inducted in $5.2 \%$ of the MPA patients and $4.1 \%$ of the GPA patients. The addition of plasma exchange
\end{abstract}

\footnotetext{
K. Sugiyama $\cdot$ K. Sada $(\bowtie) \cdot J$. Wada $\cdot$ H. Makino Department of Medicine and Clinical Science, Okayama University Graduate School of Medicine, Dentistry and Pharmaceutical Sciences, 2-5-1 Shikata-cho, Kita-ku, Okayama 700-8558, Japan

e-mail: sadakenn@md.okayama-u.ac.jp

M. Kurosawa

Department of Epidemiology and Environmental Health, Juntendo University School of Medicine, 2-1-1 Hongo, Bunkyo-ku, Tokyo, Japan
}

was associated with elevation of the serum creatinine level in patients with both MPA and GPA.

Conclusion A dominance of MPA and a reduced frequency of renal involvement in GPA patients may be significant features of the Japanese population. Clinical practice relating to MPA and GPA in Japan can be characterized as follows: CY is used less commonly, and plasma exchange is employed for patients with deteriorated renal function.

Keywords Anti-neutrophil cytoplasmic antibody (ANCA) associated vasculitis (AAV) - Cyclophosphamide . Microscopic polyangiitis (MPA) - Plasma exchange · Granulomatosis with polyangiitis (GPA)

\section{Introduction}

Microscopic polyangiitis (MPA) and granulomatosis with polyangiitis (GPA) are the most common forms of antineutrophil cytoplasmic antibody (ANCA) associated diseases characterized by necrotizing small-vessel vasculitis (AAV). Approximately $90 \%$ of patients with active, generalized MPA and GPA have circulating ANCA, and such a close association raises the possibility of pathogenic roles for ANCA. The primary antigenic targets of ANCA are myeloperoxidase (MPO-ANCA) and proteinase-3 (PR3ANCA) of granulocytes and monocytes [1]. MPA is characterized by the frequent association of MPO-ANCA and pauci-immune necrotizing vasculitis without granulomatous lesions, while GPA is characterized by a high rate of positivity for PR3-ANCA and granuloma formation in various affected organs [2].

A previous report showed that the prevalence of MPA is higher than that of GPA in Japanese patients with renal 
vasculitis, while GPA is much more common in Europe [3]. It has also been reported that older Japanese patients are more likely to be affected than European patients, and that there are significant differences in the profile of ANCA positivity. Although this report demonstrated the features of Japanese patients with renal vasculitis, the characteristics and status of Japanese patients with systemic vasculitis have been not clarified. In a recommendation from EULAR, a combination therapy including cyclophosphamide (CY) and corticosteroids for inducing remission of systemic vasculitis and the need to reduce the dose according to renal function and age were proposed [4]. However, it is not known how often CY is used and what the major determinant is for the usage of $\mathrm{CY}$ in Japanese AAV patients in clinical practice.

In Japan, MPA and GPA have been recognized and officially certified as "intractable diseases" by the Ministry of Health, Labour and Welfare (MHLW). The patients who have MPA and GPA and wish to receive public financial aid from the MHLW to cover their medical costs must sign agreements and submit applications. Patients certified according to the MHLW diagnostic criteria for MPA or GPA [5-7] are registered in the MHLW database and information about them (clinical characteristics, medical history, organ involvements, laboratory data, and treatment modalities, are stored digitally by prefectural administrations and the MHLW.

We performed this study to survey the characteristics of Japanese patients with MPA and GPA based on a data set from the MHLW. We also attempted to investigate the frequency of the concomitant use of CY or plasma exchange with corticosteroids, and determine the factors that contributed to the choice of therapies for MPA and GPA.

\section{Patients and methods}

\section{Studied patients}

We were permitted to use data on 1320 patients $(\mathrm{MPA}=988, \mathrm{GPA}=332)$ who were registered in the MHLW database from 2006 to 2008 and whose clinical data input was completed electronically. Among those 1320 patients, we identified $1032(\mathrm{MPA}=787, \mathrm{GPA}=246)$ who had registered within a year after onset. We eventually used the data for the $938(\mathrm{MPA}=697, \mathrm{GPA}=241)$ patients who fulfilled the MHLW diagnostic criteria. The criteria for MPA indicate the following three symptoms: rapidly progressive glomerulonephritis (RPGN), pulmonary hemorrhage, and other symptoms, including purpura, subcutaneous hemorrhage, gastrointestinal bleeding, and mononeuritis multiplex. Patients were certified as definite
MPA cases if (1) they were positive for two or more of the symptoms, and had positive histological findings, or (2) they were positive for two or more of the symptoms, including RPGN and pulmonary hemorrhage, and positive for MPOANCA. Probable MPA cases were (1) positive for three of the symptoms or (2) positive for one of the symptoms as well as MPO-ANCA [6]. Among the 697 MPA patients, 294 were diagnosed as being definite cases and 403 were possible cases. The criteria for GPA included nose and throat (E), lung (L), and kidney (K) symptoms, as well as others due to vasculitis. Definite GPA cases were (1) positive for three or more of the symptoms, including E, L, and K symptoms, (2) positive for two or more of the symptoms and had positive histological findings, or (3) positive for one or more of the symptoms, had positive histological findings, and were positive for PR3-ANCA/C-ANCA [7]. Among the 241 GPA patients, 168 were diagnosed as definite cases and 73 were possible cases.

\section{Data collection and arrangement}

The following information was extracted from database: sex, age, presence of a histological examination, histological findings, organ symptoms, ANCA positivity, levels of serum creatinine $(\mathrm{Cr})$ and $\mathrm{C}$-reactive protein $(\mathrm{CRP})$, corticosteroid dosage, and concomitant usage of steroid pulse therapy, CY, and plasma exchange or hemodialysis. We categorized organ symptoms into nine groups according to the BVAS scoring system [8]: "systemic symptoms," "cutaneous symptoms," "mucous membranes and eye symptoms," "ear, nose, and throat symptoms," "chest symptoms," "cardiovascular symptoms," "abdominal symptoms," "renal symptoms," and "nervous system symptoms." We sorted each item in the database into these categories. For example, we defined the existence of systemic symptoms as at least one "yes" among the following items: fever of $38{ }^{\circ} \mathrm{C}$ or higher for two weeks or longer, body weight loss of $6 \mathrm{~kg}$ or more for six months, myalgia/ myositis, and arthralgia/arthritis.

Statistical analysis

After descriptive analysis of the characteristics and treatment statuses of the MPA and GPA patients, we compared the characteristics of the patients who concomitantly use $\mathrm{CY}$ and those who used corticosteroid alone. We calculated the estimated glomerular filtration rate (eGFR) using the modification of diet in renal disease (MDRD) equation: $194.9 \times$ serum $\mathrm{Cr}^{-1.094 .9} \times$ age $^{-0.287}(\times 0.739$ if female $)$ [9]. We categorized the patients into five groups according to eGFR and evaluated the correlation of these groups with CY usage for MPA and GPA by ANOVA. Similarly, in each disease category, we separated the patients into 
subgroups who did undergo plasma exchange and those who did not, and compared their characteristics. In order to identify the independent factors associated with concomitant usage of $\mathrm{CY}$, the variables extracted in the univariate analysis were entered into a multivariate analysis using a logistic regression model. All of the statistical analyses in this study were performed using the statistical package of the program JMP for Windows, version 8.0 (SAS Institute Inc., Cary, NC, USA). Clinical variables that may have been related to the outcomes were compared by MannWhitney $U$ and chi-square tests (univariate model). All results were expressed as the mean $\pm \mathrm{SE}$ (standard error), and statistical significance was defined as a $p$ value of less than 0.05 (two-tailed).

\section{Results}

Patient characteristics of MPA and GPA

We identified 697 MPA patients and 241 GPA patients. Patient characteristics are shown in Table 1. The mean age of the MPA patients was $69.4 \pm 0.4$ years, and that of the GPA patients was $58.4 \pm 1.1$ years. Renal involvement was most frequently observed in MPA patients $(86.9 \%)$, while ear, nose, and throat (ENT) symptoms were most common in GPA patients (86.7 \%). Other major symptoms were systemic $(80.3 \%)$, chest $(73.7 \%)$, and nervous system $(45.2 \%)$ involvement in MPA patients, while systemic $(81.3 \%)$, chest $(78.0 \%)$, and renal $(60.6 \%)$ involvement were frequently noted in GPA patients. MPO-ANCA or p-ANCA was positive in $97.1 \%$ of the MPA patients and PR3-ANCA or c-ANCA was positive in $73.0 \%$ of the GPA patients. All but five patients with MPA and seven patients with GPA were treated with corticosteroids, and the mean maximum daily dosage of prednisolone was $26.5 \pm 0.9 \mathrm{mg} /$ day in the MPA patients and $35.3 \pm 1.6$ $\mathrm{mg} /$ day in the GPA patients. CY was used concomitantly by $22.2 \%$ of the MPA patients and $58.5 \%$ of the GPA patients. Plasma exchanges were performed in 5.2 and $4.1 \%$ of the MPA and GPA patients, respectively.

Characteristics of MPA and GPA patients treated with $\mathrm{CY}$ or corticosteroid alone

Both MPA and GPA patients treated with CY were significantly younger than those treated with corticosteroid alone $(66.1 \pm 0.9$ vs. $70.1 \pm 0.5$ years, $p=0.0002$, Table 2 ; and $57.2 \pm 1.4$ vs. $62.0 \pm 1.8$ years, $p=0.0395$, Table 3, respectively). Concomitant use of CY was seen significantly less frequently in GPA patients with lower eGFR (Fig. 1, $p=0.014$ ), but not significantly less frequently in such MPA patients (Fig. $1, p=0.370$ ). GPA
Table 1 Characteristics of the MPA and GPA patients

\begin{tabular}{|c|c|c|}
\hline & $\begin{array}{l}\text { MPA } \\
(n=697)\end{array}$ & $\begin{array}{l}\text { GPA } \\
(n=241)\end{array}$ \\
\hline \multicolumn{3}{|l|}{ Diagnosis } \\
\hline Definite:possible & $294: 403$ & $168: 73$ \\
\hline Men:women & 299:398 & 139:102 \\
\hline Age, mean \pm SE years & $69.4 \pm 0.4$ & $58.4 \pm 1.1$ \\
\hline \multicolumn{3}{|l|}{ Symptoms } \\
\hline Systemic symptoms (\%) & 80.3 & 81.3 \\
\hline Cutaneous symptoms (\%) & 35.4 & 26.1 \\
\hline Mucous membrane and eyes $(\%)$ & 13.1 & 46.1 \\
\hline Ear, nose, and throat $(\%)$ & 14.1 & 86.7 \\
\hline Chest $(\%)$ & 73.7 & 78.0 \\
\hline Cardiovascular $(\%)$ & 14.3 & 15.8 \\
\hline Abdominal (\%) & 10.2 & 7.1 \\
\hline Renal (\%) & 86.9 & 60.6 \\
\hline Nervous system (\%) & 45.2 & 32.3 \\
\hline Pulmonary hemorrhage $(\%)$ & 11.3 & ND \\
\hline $\begin{array}{l}\text { Rapidly progressive } \\
\text { glomerulonephritis (\%) }\end{array}$ & 63.2 & 25.7 \\
\hline \multicolumn{3}{|l|}{ Examination } \\
\hline $\mathrm{Cr}$, mean $\pm \mathrm{SE}(\mathrm{mg} / \mathrm{dl})$ & $2.5 \pm 0.1$ & $1.6 \pm 0.2$ \\
\hline eGFR (ml/min/1.73 $\left.\mathrm{m}^{2}\right)$ & $43.2 \pm 1.3$ & $75.3 \pm 3.2$ \\
\hline $\mathrm{CRP}$, mean $\pm \mathrm{SE}(\mathrm{mg} / \mathrm{dl})$ & $9.0 \pm 0.3$ & $10.2 \pm 0.5$ \\
\hline MPO- or p-ANCA positive $(\%)$ & 97.1 & 17.4 \\
\hline PR3- or c-ANCA positive $(\%)$ & 7.0 & 73.0 \\
\hline Histological finding & 30.6 & 52 \\
\hline \multicolumn{3}{|l|}{ Treatment } \\
\hline $\begin{array}{l}\text { Max. oral PSL dosage, mean } \pm \text { SE } \\
\text { mg/day }\end{array}$ & $26.5 \pm 0.9$ & $35.3 \pm 1.6$ \\
\hline m-PSL pulse $(\%)$ & 51.8 & 38.2 \\
\hline Immunosuppressants usage $(\%)$ & 27.8 & 64.3 \\
\hline CY usage $(\%)$ & 22.2 & 58.5 \\
\hline Plasma exchange (\%) & 5.2 & 4.1 \\
\hline Dialysis treatment $(\%)$ & 11.9 & 6.6 \\
\hline
\end{tabular}

$C Y$ cyclophosphamide, $S E$ standard error, $C r$ creatinine, $C R P$ C-reactive protein, $e G F R$ estimated glomerular filtration rate, $M P O$ ANCA myeloperoxidase anti-neutrophil cytoplasmic antibody, $p$-ANCA perinuclear anti-neutrophil cytoplasmic antibody, PR3$A N C A$ proteinase-3 anti-neutrophil cytoplasmic antibody, $c$-ANCA cytoplasmic anti-neutrophil cytoplasmic antibody, $P S L$ prednisolone, $m$-PSL methyl prednisolone

patients with RPGN or nervous system symptoms were treated with $\mathrm{CY}$ less frequently than with corticosteroid alone. In contrast, MPA patients with cutaneous symptoms or pulmonary hemorrhage and GPA patients with ENT symptoms were treated with $\mathrm{CY}$ more frequently. The multivariate analysis included the variables extracted in the univariate analysis. Age and pulmonary hemorrhage in MPA and nervous system symptoms and RPGN in GPA were independent factors for the concomitant usage of $\mathrm{CY}$ (Table 4). The mean maximum daily dose of prednisone 
Table 2 Characteristics of MPA patients treated with concomitant cyclophosphamide and corticosteroid monotherapy

$C Y$ cyclophosphamide, $C S$ corticosteroids, $S E$ standard error, $\mathrm{Cr}$ creatinine, $e G F R$ estimated glomerular filtration rate, $C R P$ C-reactive protein, $M P O-A N C A$ myeloperoxidase anti-neutrophil cytoplasmic antibody, $p-A N C A$ perinuclear anti-neutrophil cytoplasmic antibody, PR3-ANCA proteinase-3 anti-neutrophil cytoplasmic antibody, $c$-ANCA cytoplasmic anti-neutrophil cytoplasmic antibody, $P S L$ prednisolone, $m$-PSL methyl prednisolone

$* p<0.05$

Table 3 Characteristics of GPA patients treated with concomitant cyclophosphamide and corticosteroid monotherapy
$C Y$ cyclophosphamide, $C S$ corticosteroids, $S E$ standard error, $\mathrm{Cr}$ creatinine, $e G F R$ estimated glomerular filtration rate, CRP C-reactive protein, $P S L$ prednisolone, $m-P S L$ methyl prednisolone

\begin{tabular}{llll}
\hline & $\begin{array}{l}\text { Concomitant CY usage } \\
(n=155)\end{array}$ & $\begin{array}{l}\text { CS monotherapy } \\
(n=503)\end{array}$ & $p$ \\
\hline Age, mean \pm SE years & $66.1 \pm 0.9$ & $70.1 \pm 0.5$ & $0.0002^{*}$ \\
Symptoms & & & \\
Systemic (\%) & 81.9 & 80.0 & 0.6440 \\
Cutaneous (\%) & 43.2 & 32.0 & $0.0120^{*}$ \\
Mucous membrane and eyes (\%) & 11.6 & 13.0 & 0.7823 \\
Ear, nose, and throat (\%) & 15.5 & 13.4 & 0.5083 \\
Chest (\%) & 74.8 & 73.0 & 0.6785 \\
Cardiovascular $(\%)$ & 11.0 & 14.8 & 0.2873 \\
Abdominal (\%) & 12.9 & 9.4 & 0.2252 \\
Renal (\%) & 85.8 & 87.2 & 0.6833 \\
Nervous system (\%) & 45.8 & 44.2 & 0.7815 \\
Pulmonary hemorrhage $(\%)$ & 18.1 & 8.2 & $0.0009 *$ \\
Rapidly progressive glomerulonephritis $(\%)$ & 59.4 & 64.8 & 0.2518 \\
Cr, mean \pm SE mg/dl & $2.1 \pm 0.3$ & $2.7 \pm 0.2$ & 0.0635 \\
eGFR (ml/min/1.73 m $\left.{ }^{2}\right)$ & $48.0 \pm 2.7$ & $41.8 \pm 1.5$ & $0.0452^{*}$ \\
CRP, mean \pm SE mg/dl & $9.6 \pm 0.6$ & $8.9 \pm 0.3$ & 0.2763 \\
Max. oral PSL dosage, mean \pm SE mg/day & $32.6 \pm 1.9$ & $25.2 \pm 1.0$ & $0.0008^{*}$ \\
m-PSL pulse (\%) & 58.1 & 50.0 & 0.0813 \\
Plasma exchange (\%) & 9.7 & 3.8 & $0.0066^{*}$ \\
Dialysis treatment $(\%)$ & 9.0 & 12.2 & 0.3146 \\
\hline
\end{tabular}

\begin{tabular}{llll}
\hline & $\begin{array}{l}\text { Concomitant CY usage } \\
(n=141)\end{array}$ & $\begin{array}{l}\text { CS monotherapy } \\
(n=86)\end{array}$ & $p$ \\
\hline Age, mean \pm SE years & $57.2 \pm 1.4$ & $62.0 \pm 1.8$ & $0.0395^{*}$ \\
Symptoms & & & \\
Systemic (\%) & 83.7 & 75.0 & 0.1566 \\
Cutaneous (\%) & 26.2 & 26.3 & 1.000 \\
Mucous membrane and eyes (\%) & 46.8 & 43.8 & 0.6760 \\
Ear, nose, and throat (\%) & 90.1 & 80.0 & $0.0421^{*}$ \\
Chest (\%) & 80.9 & 75.0 & 0.3106 \\
Cardiovascular $(\%)$ & 13.5 & 23.8 & 0.0637 \\
Abdominal $(\%)$ & 6.4 & 7.5 & 0.7846 \\
Renal (\%) & 59.6 & 61.3 & 0.8865 \\
Nervous system (\%) & 27.0 & 45.0 & $0.0077^{*}$ \\
Rapidly progressive & 19.9 & 37.5 & $0.0065^{*}$ \\
glomerulonephritis $(\%)$ & & & \\
Cr, mean \pm SE mg/dl & $1.5 \pm 0.2$ & $1.8 \pm 0.3$ & 0.4520 \\
eGFR (ml/min/1.73 m $\left.{ }^{2}\right)$ & $80.2 \pm 3.9$ & $63.3 \pm 5.3$ & $0.0106^{*}$ \\
CRP, mean \pm SE mg/dl & $10.6 \pm 0.7$ & $9.3 \pm 0.9$ & 0.2039 \\
Max. PSL dosage, mean \pm SE mg/day & $39.3 \pm 2.0$ & $28.7 \pm 2.7$ & $0.0018^{*}$ \\
m-PSL pulse (\%) & 39.0 & 41.3 & 0.7760 \\
Plasma exchange $(\%)$ & 5.0 & 2.5 & 0.4933 \\
Dialysis treatment $(\%)$ & 5.0 & 10.0 & 0.1711 \\
\hline
\end{tabular}

for the patients treated with corticosteroid plus CY was significant higher than the patients treated with corticosteroid alone in both MPA patients (32.6 \pm 1.9 vs. $25.2 \pm$ $1.0 \mathrm{mg} / \mathrm{day}, p=0.0008)$ and GPA patients $(39.4 \pm 2.1 \mathrm{vs}$. $29.5 \pm 2.5 \mathrm{mg} /$ day, $p=0.0025$ ).
Patient characteristics of MPA and GPA treated with or without plasma exchange

The mean ages of the patients who underwent plasma exchange and those who did not were similar for both the 




Fig. 1 The percentage of the patients in each category of eGFR who were treated with corticosteroid plus CY. $C Y$ cyclophosphamide, $e G F R$ estimated glomerular filtration rate, ${ }^{*} p<0.05$

Table 4 Logistic regression analysis of the independent factors associated with concomitant $\mathrm{CY}$ use

\begin{tabular}{lll}
\hline Variable & Odds ratio $(95 \% \mathrm{CI})$ & $p$ \\
\hline MPA & & \\
Age (per yr) & $0.97(0.96-0.99)$ & $0.0025^{*}$ \\
Pulmonary hemorrhage & $2.76(1.61-4.71)$ & $0.0003^{*}$ \\
eGFR (ml/min/1.73 m²) & $1.00(1.00-1.01)$ & 0.1660 \\
Cutanous & $1.40(0.94-2.09)$ & 0.0986 \\
GPA & & \\
Age (per year) & $0.99(0.97-1.00)$ & 0.1889 \\
ENT symptoms & $1.96(0.85-4.60)$ & 0.1151 \\
Nervous system symptoms & $0.48(0.26-0.88)$ & $0.0174^{*}$ \\
RPGN & $0.45(0.24-0.87)$ & $0.0165^{*}$ \\
\hline
\end{tabular}

$E N T$ ear, nose, and throat, $e G F R$ estimated glomerular filtration rate, $R P G N$ rapidly progressive glomerulonephritis

$* p<0.05$

MPA $\quad(68.6 \pm 1.9$ vs. $69.4 \pm 0.5$ years, $p=0.7041$; Table 5) and the GPA (57.9 \pm 5.3 vs. $58.5 \pm 1.1$ years, $p=0.9162$; Table 6) patients. The MPA patients who were treated with plasma exchange had pulmonary hemorrhages more frequently than those who were not (18.1 vs. $8.2 \%$, $p=0.0009$ ). The serum levels of $\mathrm{Cr}$ in patients who underwent plasma exchange were higher than the levels in those who did not undergo plasma exchange for both the MPA ( $3.8 \pm 0.5$ vs. $2.4 \pm 0.1 \mathrm{mg} / \mathrm{dl}, p=0.0132)$ and the GPA $(3.7 \pm 0.8$ vs. $1.5 \pm 0.2, p=0.0102)$ patients. Furthermore, the patients treated with plasma exchange had RPGN more frequently than those who were not treated with it among GPA patients, and all of the GPA patients who underwent plasma exchange had renal symptoms. Similarly, dialysis treatment was more frequent in the patients who underwent plasma exchange than in those who did not among MPA patients (50.0 vs. 9.8\%, $p<0.0001)$ and among GPA patients (60.0 vs. $4.3 \%$, $p<0.0001)$.
Table 5 Characteristics of MPA patients who did and did not undergo plasma exchange

\begin{tabular}{|c|c|c|c|}
\hline & $\begin{array}{l}\mathrm{PE} \\
(n=36)\end{array}$ & $\begin{array}{l}\text { Non-PE } \\
(n=661)\end{array}$ & $p$ \\
\hline Age, mean \pm SE years & $68.6 \pm 1.9$ & $69.4 \pm 0.5$ & 0.7041 \\
\hline \multicolumn{4}{|l|}{ Symptoms } \\
\hline Systemic (\%) & 69.4 & 80.9 & 0.1280 \\
\hline Cutaneous (\%) & 44.4 & 35.0 & 0.2835 \\
\hline $\begin{array}{l}\text { Mucous membrane and } \\
\text { eyes }(\%)\end{array}$ & 27.8 & 12.3 & $0.0178^{*}$ \\
\hline Ear, nose, and throat (\%) & 16.7 & 13.9 & 0.6229 \\
\hline Chest $(\%)$ & 83.3 & 73.2 & 0.2426 \\
\hline Cardiovascular $(\%)$ & 25.0 & 13.8 & 0.0827 \\
\hline Abdominal (\%) & 8.3 & 10.3 & 1.0000 \\
\hline Renal (\%) & 97.2 & 86.4 & 0.0722 \\
\hline Nervous system (\%) & 33.3 & 45.8 & 0.1697 \\
\hline Pulmonary hemorrhage $(\%)$ & 36.1 & 10.0 & $<0.0001 *$ \\
\hline $\begin{array}{l}\text { Rapidly progressive } \\
\text { glomerulonephritis (\%) }\end{array}$ & 72.2 & 62.8 & 0.2902 \\
\hline $\mathrm{Cr}$, mean $\pm \mathrm{SE} \mathrm{mg} / \mathrm{dl}$ & $3.8 \pm 0.5$ & $2.4 \pm 0.1$ & $0.0132 *$ \\
\hline $\mathrm{eGFR}\left(\mathrm{ml} / \mathrm{min} / 1.73 \mathrm{~m}^{2}\right)$ & $26.7 \pm 5.7$ & $44.1 \pm 1.3$ & $0.0031 *$ \\
\hline $\mathrm{CRP}$, mean \pm SE mg/dl & $12.2 \pm 1.1$ & $8.8 \pm 0.3$ & $0.0037 *$ \\
\hline $\begin{array}{c}\text { Max. oral PSL dosage, } \\
\text { mean } \pm \text { SE mg/day }\end{array}$ & $23.5 \pm 4.2$ & 26.7. \pm 0.9 & 0.4580 \\
\hline m-PSL pulse $(\%)$ & 86.1 & 49.9 & $<0.0001^{*}$ \\
\hline CY usage (\%) & 41.7 & 21.2 & $0.0068 *$ \\
\hline Dialysis treatment $(\%)$ & 50.0 & 9.8 & $<0.0001 *$ \\
\hline
\end{tabular}

$C Y$ cyclophosphamide, $S E$ standard error, $C r$ creatinine, $e G F R$ estimated glomerular filtration rate, $C R P$ C-reactive protein, $P S L$ prednisolone, $m$-PSL methyl prednisolone

$* p<0.05$

\section{Discussion}

We determined the characteristics and the current treatment statuses of MPA and GPA patients based on a data set from the MHLW database in Japan. In the present study, the ratio of the patients with MPA to the patients with GPA was 3:1. Watts et al. [10] reported that the ratio of MPA patients to GPA patients was 3:4 in the UK. The predominance of MPA compared with GPA in Japan seen in our study was similar to that noted in a previous report $[3,11]$. MPO-ANCA- or p-ANCA-positive MPA patients and PR3ANCA- or c-ANCA-positive GPA patients were more common in this study. These results are consistent with a previous Japanese nationwide epidemiological survey which showed that $87.3 \%$ of MPA patients were p-ANCA positive and $85.7 \%$ of WG patients were c-ANCA positive [11]. A report on a Western patient population showed that $30.4 \%$ of MPA patients were MPO-ANCA or p-ANCA positive and $57.4 \%$ of GPA patients were PR3-ANCA or c-ANCA positive [12]. The MHLW criteria for MPA and 
Table 6 Characteristics of GPA patients who did and did not undergo plasma exchange

\begin{tabular}{|c|c|c|c|}
\hline & $\begin{array}{l}\mathrm{PE} \\
(n=10)\end{array}$ & $\begin{array}{l}\text { Non-PE } \\
(n=231)\end{array}$ & $p$ \\
\hline Age, mean \pm SE years & $57.9 \pm 5.3$ & $58.5 \pm 1.1$ & 0.9162 \\
\hline \multicolumn{4}{|l|}{ Symptoms } \\
\hline Systemic (\%) & 80.0 & 81.4 & 1.000 \\
\hline Cutaneous (\%) & 40.0 & 25.5 & 0.2931 \\
\hline $\begin{array}{l}\text { Mucous membrane and } \\
\text { eyes }(\%)\end{array}$ & 40.0 & 46.3 & 0.7566 \\
\hline Ear, nose, and throat (\%) & 80.0 & 87.0 & 0.6262 \\
\hline Chest $(\%)$ & 90.0 & 77.5 & 0.6958 \\
\hline Cardiovascular (\%) & 40.0 & 14.7 & 0.0547 \\
\hline Abdominal (\%) & 10.0 & 6.9 & 0.5258 \\
\hline Renal (\%) & 100.0 & 58.9 & $0.0071^{*}$ \\
\hline Nervous system (\%) & 50.0 & 31.6 & 0.3000 \\
\hline $\begin{array}{l}\text { Rapidly progressive } \\
\text { glomerulonephritis (\%) }\end{array}$ & 60.0 & 24.2 & $0.0203^{*}$ \\
\hline $\mathrm{Cr}$, mean $\pm \mathrm{SE} \mathrm{mg} / \mathrm{dl}$ & $3.7 \pm 0.8$ & $1.5 \pm 0.2$ & $0.0102^{*}$ \\
\hline eGFR $\left(\mathrm{ml} / \mathrm{min} / 1.73 \mathrm{~m}^{2}\right)$ & $33.1 \pm 15.5$ & $77.1 \pm 3.2$ & $0.0058^{*}$ \\
\hline $\mathrm{CRP}$, mean $\pm \mathrm{SE} \mathrm{mg} / \mathrm{dl}$ & $11.2 \pm 2.6$ & $10.2 \pm 0.5$ & 0.7008 \\
\hline $\begin{array}{c}\text { Max. oral PSL dosage, } \\
\text { mean } \pm \text { SD mg/day }\end{array}$ & $20.0 \pm 8.4$ & $35.9 \pm 1.6$ & 0.0663 \\
\hline m-PSL pulse $(\%)$ & 90.0 & 35.9 & $0.0009^{*}$ \\
\hline CY usage $(\%)$ & 70.0 & 57.6 & 0.5268 \\
\hline Dialysis treatment $(\%)$ & 60.0 & 4.3 & $<0.0001^{*}$ \\
\hline
\end{tabular}

$P E$ plasma exchange, $S E$ standard error, $C r$ creatinine, $e G F R$ estimated glomerular filtration rate, $C R P$ C-reactive protein, $P S L$ prednisolone, $m$-PSL methyl prednisolone

$* p<0.05$

GPA consist of three parts: clinical symptoms, histological findings, and ANCA positivity. The MPA criteria include MPO-ANCA or p-ANCA positivity while the GPA criteria include PR3-ANCA or c-ANCA positivity. There is no reference to ANCA in the classification criteria of the American College of Rheumatology (ACR) or the Chapel Hill Consensus Conference. Although Watts et al. included ANCA positivity in their classification algorithm, the type of ANCA was not taken into account in the diagnosis of MPA and GPA. These differences between the MHLW criteria and other criteria could have affected the numbers of ANCA-positive GPA and MPA patients in our study. In addition, the MHLW criteria emphasize specific clinical symptoms, such as RPGN and pulmonary hemorrhage, meaning that our study population may differ from those in previous reports. Renal involvement was noted in only $60 \%$ of patients with GPA in the present study, even if all renal symptoms were included; glomerulonephritis occurred in almost $80 \%$ of GPA patients during their clinical courses in the USA [2]. Only patients who had registered within a year after disease onset were included in this study. In addition, the MHLW criteria for possible GPA cases comprise fewer clinical symptoms than the ACR criteria for diagnosing GPA. Therefore, more organ-limited patients may be present in this study population.

Concomitant CY usage was a less common option for treatment of AAV in the present study. Only $22 \%$ of the patients with MPA and $59 \%$ of the patients with GPA were treated with $\mathrm{CY}$ combined with corticosteroids, despite the fact that several guidelines recommend the concomitant usage of CY for AAV [4, 13]. Indeed, $89 \%$ of the AAV patients were treated with $\mathrm{CY}$ combined with corticosteroids according to a previous report on a European population [14]. Even in another Asian country, $94 \%$ of the MPA patients were treated with a combination of $\mathrm{CY}$ and corticosteroids [15]. A previous nationwide survey of the Japanese population showed that $\mathrm{CY}$ was used by $62.3 \%$ of MPA and $96.3 \%$ of GPA patients [11], and a recent study of Japanese patients with MPO-ANCA-associated vasculitis (JMAAV) also reported that $58.3 \%$ of the patients were treated with CY [16]. Therefore, less common usage of $\mathrm{CY}$ may be a characteristic of the current Japanese therapy for AAV. In the multivariate analysis, avoidance of concomitant CY usage was associated with older age and pulmonary hemorrhage in MPA patients, and RPGN and nervous system symptoms in GPA patients. Several guidelines recommend that the dosage of CY should be reduced for elderly patients or those with deteriorated renal function in order to avoid adverse effects $[4,13]$. Additionally, a Japanese nationwide survey of RPGN reported that there was no additional benefit of immunosuppressants for the renal prognoses of elderly patients based on data for 715 RPGN patients collected until 2001 [17]. Therefore, avoidance of concomitant usage rather than proactive usage with the dose of $\mathrm{CY}$ reduced for elderly patients and patients with RPGN may be a significant feature of the current treatment approach for MPA in Japan.

The maximum daily dose of corticosteroids was higher in patients who concomitantly used CY than in those who did not. During the treatment of AAV patients, it is common practice for the initial dose of corticosteroids to be continued for one month, whereas a more rapidly tapering regimen of corticosteroids was adopted in several recent clinical trials (patients initially received $1 \mathrm{mg} / \mathrm{kg} /$ day of oral prednisolone, which was reduced to $0.75 \mathrm{mg} / \mathrm{kg} / \mathrm{day}$ after one week, and $0.50 \mathrm{mg} / \mathrm{kg} /$ day after two weeks) [18-20]. Because the body weights of patients with AAV were not registered in the database, we were not able to convert the daily dose of corticosteroids per body into the dose per kilogram in this study. However, AAV patients are commonly treated with about $0.8 \mathrm{mg} / \mathrm{kg} / \mathrm{day}$ of oral prednisolone in Japan. Although the rate of tapering of corticosteroids could not be assessed in present study, 
patients who are concomitantly using CY may be treated with a higher dose of corticosteroid and tapered more rapidly, as in the regimen applied in recent clinical trials.

In our study, about $5 \%$ of the patients were treated with plasma exchange. This result is similar to that seen in the JMAAV study, which reported that plasma exchange was applied in only 2 of 48 patients [16]. A recent Western study of remission maintenance showed that almost $15 \%$ of patients with AAV were treated with plasma exchange, and the median level of serum $\mathrm{Cr}$ was 2.9 or $2.7 \mathrm{mg} / \mathrm{dl}$ [18]. The addition of plasma exchange was associated with an elevation in the serum creatinine level in both MPA and GPA patients. Plasma exchange may be initiated based on renal dysfunction rather than disease classification. The European randomized Methylprednisolone versus Plasma Exchange (MEPEX) trial showed that plasma exchange increased the rate of renal recovery in ANCA-associated systemic vasculitis that presented with renal failure [20]. The EULAR recommendations propose the concomitant usage of plasma exchange for patients with rapidly progressive severe renal disease based on this clinical trial [4]. Because of the less severe deterioration in renal function observed in our population, the concomitant usage of plasma exchange may have been less common. Among all chest symptoms, plasma exchange was performed most commonly in patients with pulmonary hemorrhage in our study. The British Society for Rheumatology and British Health Professionals in Rheumatology guidelines for vasculitis recommend that treatment with plasma exchange should be considered in patients with life-threatening manifestations of disease such as pulmonary hemorrhage as well as in patients presenting with severe renal failure [13].

Several limitations of the present study should be noted. First, we were not able to reconfirm the clinical data by checking medical records. Sakauchi et al. [21], who studied the etiology of primary biliary cirrhosis using the MHLW database, noted a similar limitation. In addition, we cannot discuss the reliability of the data used in the present study since this is the first report on the characteristics of MPA and GPA patients in Japan. Secondly, we applied the MHLW criteria to diagnose MPA and GPA patients in the present study; however, the specificity and sensitivity of the MHLW criteria have not yet been validated. Third, the choice of therapeutic modality may be influenced by healthcare access. Finally, our investigation failed to demonstrate an association between these treatments and prognosis since it was a cross-sectional study.

In conclusion, the dominance of MPA patients over GPA patients and a lower frequency of renal involvement in GPA patients may be significant features of Japanese MPA and GPA patients. Concomitant CY usage was relatively less commonly used to treat AAV in Japan. Plasma exchange was used in AAV patients with deteriorated renal function.
Further investigations based on global definitions are required to further confirm these features of Japan patients.

Acknowledgments The authors thank all of the physicians who supported the nationwide surveys of MPA and GPA patients in Japan. This work was supported in part by grants from the Ministry of Health, Labour and Welfare, Japan.

Open Access This article is distributed under the terms of the Creative Commons Attribution License which permits any use, distribution, and reproduction in any medium, provided the original author(s) and the source are credited.

\section{References}

1. Franssen CF, Stegeman CA, Kallenberg CG, Gans RO, De Jong PE, Hoorntje SJ, et al. Antiproteinase 3- and antimyeloperoxidaseassociated vasculitis. Kidney Int. 2000;57(6):2195-206.

2. Duna GF, Galperin C, Hoffman GS. Wegener's granulomatosis. Rheum Dis Clin North Am. 1995;21(4):949-86.

3. Watts RA, Scott DG, Jayne DR, Ito-Ihara T, Muso E, Fujimoto S, et al. Renal vasculitis in Japan and the UK-are there differences in epidemiology and clinical phenotype? Nephrol Dial Transplant. 2008;23(12):3928-31.

4. Mukhtyar C, Guillevin L, Cid MC, Dasgupta B, de Groot K, Gross W, et al. EULAR recommendations for the management of primary small and medium vessel vasculitis. Ann Rheum Dis. 2009;68(3):310-7.

5. Ozaki S. ANCA-associated vasculitis: diagnostic and therapeutic strategy. Allergol Int. 2007;56(2):87-96.

6. Nakabayashi K, Hashimoto H (editors) Microscopic polyangiitis. Tokyo: Research Group of Intractable Vasculitis, Ministry of Health, Labor, and Welfare of Japan; 2002.

7. Yoshida M (editor) Wegener's granulomatosis. Tokyo: Research Group of Intractable Vasculitis, Ministry of Health, Labor, and Welfare of Japan; 2002.

8. Luqmani RA, Bacon PA, Moots RJ, Janssen BA, Pall A, Emery $\mathrm{P}$, et al. Birmingham Vasculitis Activity Score (BVAS) in systemic necrotizing vasculitis. QJM. 1994;87(11):671-8.

9. Matsuo S, Imai E, Horio M, Yasuda Y, Tomita K, Nitta K, et al. Revised equations for estimated GFR from serum creatinine in Japan. Am J Kidney Dis. 2009;53(6):982-92.

10. Watts RA, Lane SE, Bentham G, Scott DG. Epidemiology of systemic vasculitis: a ten-year study in the United Kingdom. Arthritis Rheum. 2000;43(2):414-9.

11. Hashimoto H, Yoshida M, Kobayashi S, Eishi K, Tsusaka N, Nakabayashi K, et al. An epidemiologic nationwide survey of ANCA related vasculitis in Japan.: annual report 1998 of the Research Group for Intractable Vasculitis, Ministry of Health, Labor, and Welfare of Japan. Tokyo: Research Group of Intractable Vasculitis, Ministry of Health, Labor, and Welfare of Japan; 1999, pp 213-29.

12. Lane SE, Watts RA, Shepstone L, Scott DG. Primary systemic vasculitis: clinical features and mortality. QJM. 2005;98(2): 97-111.

13. Lapraik C, Watts R, Bacon P, Carruthers D, Chakravarty K, D'Cruz D, et al. BSR and BHPR guidelines for the management of adults with ANCA associated vasculitis. Rheumatology (Oxford). 2007;46(10):1615-6.

14. Lurati-Ruiz F, Spertini F. Predictive value of antineutrophil cytoplasmic antibodies in small-vessel vasculitis. J Rheumatol. 2005;32(11):2167-72. 
15. Oh JS, Lee CK, Kim YG, Nah SS, Moon HB, Yoo B. Clinical features and outcomes of microscopic polyangiitis in Korea. J Korean Med Sci. 2009;24(2):269-74.

16. Ozaki S, Atsumi T, Hayashi T, Ishizu A, Kobayashi S, Kumagai $\mathrm{S}$, et al. Severity-based treatment for Japanese patients with MPO-ANCA-associated vasculitis: the JMAAV study. Mod Rheumatol. 2012;22(3):394-404.

17. Sakai H, Kurokawa K, Koyama A, Arimura Y, Kida H, Shigematsu S. Clinical guildeline for rapidly progressive glomerulonephritis in Japan. Jpn J Nephrol. 2002;44:55-82.

18. Hiemstra TF, Walsh M, Mahr A, Savage CO, de Groot K, Harper L, et al. Mycophenolate mofetil vs azathioprine for remission maintenance in antineutrophil cytoplasmic antibody-associated vasculitis: a randomized controlled trial. JAMA. 2010;304(21):2381-8.
19. de Groot K, Harper L, Jayne DR. Flores Suarez LF, Gregorini G, Gross WL, et al. Pulse versus daily oral cyclophosphamide for induction of remission in antineutrophil cytoplasmic antibodyassociated vasculitis: a randomized trial. Ann Intern Med. 2009;150(10):670-80.

20. Jayne DR, Gaskin G, Rasmussen N, Abramowicz D, Ferrario F, Guillevin L, et al. Randomized trial of plasma exchange or highdosage methylprednisolone as adjunctive therapy for severe renal vasculitis. J Am Soc Nephrol. 2007;18(7):2180-8.

21. Sakauchi F, Oura A, Ohnishi H, Mori M. Comparison of the clinical features of Japanese patients with primary biliary cirrhosis in 1999 and 2004: utilization of clinical data when patients applied to receive public financial aid. J Epidemiol. 2007; 17(6):210-4. 